\title{
Opposed-Piston Two-Stroke Diesel Engine Scavenging System Structural Optimization Based on GA-SVM
}

\author{
Yu-Hang LIU, Fu-Jun ZHANG*, Zhen-Feng ZHAO and Shuan-Lu ZHANG \\ Beijing Institute of Technology, Beijing, China
}

Keywords: OP2S engine, scavenging system, optimization, GA-SVM

\begin{abstract}
Opposed-Piston Two-Stroke (OP2S) diesel engines have been successfully used in many applications because of its high power efficiency. Uniform scavenging is used on OP2S diesel engines. Different from conventional diesel engine exchanging systems, intake ports and exhaust ports are located at each sides of the cylinder as OP2S scavenging system. A scavenging system optimization method, Genetic Algorithm-Support Vector Machine (GA-SVM), which employs Indicated Mean Effective Pressure (IMEP) as optimization goal is presented. The combinations of five key parameters including the width and length of intake and exhaust ports and the crank asymmetric angle are designed. All these combinations are calculated by GT-Power. The predicting model of OP2S diesel engine's IMEP is built based on the 1D results. After verifying the accuracy of the predicting model, the optimized parameters are found. The results illustrate: using 1-D simulation coupled GA-SVM to optimize scavenging parameters in OP2S diesel engines is a rapid and effective method. The optimized IMEP is 12.86 bar. This paper presents some guidelines in the further OP2S diesel engines research.
\end{abstract}

\section{Introduction}

The opposed-piston two-stroke engine (OP2S) concept came into being in 1850`s. Wittig, a German engineer, designed and manufactured the world's first coal gas fuel opposed-piston engine [1].From then on, many novel designs were brought out on aircrafts, marines and vehicles such as TS-3 engine manufactured by British Rootes company and 6-TD engine from Ukraine. However, rigid emission legislations slowed down the development of opposed-piston engines after 1970's. In recent years, with the improvement of modern analytical tools, materials, and fuel supply system, engines from FEV, EcoMotor and Achates Power company have performed well in many fields $[2,3,4,5,6]$. OP2S draw more and more attention once again because of its simple, lightweight, high efficiency and high power density [7].

In OP2S scavenging system, two pistons reciprocate opposite to each other in a common cylinder, piston port timings are controlled by two pistons. With this structural design, the need for cylinder head and valve mechanism are eliminated. In order to achieve better scavenging performance, larger ports area is wanted. However, according to the research by Peter Hofbauer [8], although larger intake and exhaust ports area can achieve better scavenging performance, shorter compression and expansion processes are inevitable. This will lead to the decrease of power efficiency. Zhenyu Zhang [9] also found larger crank asymmetric angle is benefit to the scavenging efficiency meanwhile compression ratio and expansion ratio decrease along with the increase of the crank asymmetric angle. This is also unfavorable to the development of engine performance. Therefore, in optimizing OP2S scavenging process, only taking scavenging efficiency into account is unreasonable. Thermal efficiency should also be taken into account. With the consideration of this, IMEP is selected as optimizing goal.

In engine scavenging system optimization area, two simulation approaches are widely used. The first one is Computational Fluid Dynamics (CFD) method using such as AVL-Fire, Star-CD. The CFD method can model the scavenging process with complex geometries. However, its calculation time is very long. This is unfavorable in initial engine designing. The second one is a predictive simulation method which is required to determine basic engine parameters in order to shorten the development time [10]. GT-Power is always the priority choice. Compared with detailed simulation, the 1D simulation has simpler model and it has lower demands with calculation. So 1-D simulation 
coupled with some data analysis methods can be more efficient for optimizing multi parameters in scavenging system optimization.

In data analysis area, SVM method, as a supervised learning algorithm, has been applied to many searching area with the ability of predicting. It is an outstanding statistical learning theory and especially suitable for small samples and non-linear problems [11]. However, the largest problem encountered in constructing the SVM model is how to select the parameter values of SVM. Inappropriate parameter values will leads to poor predicting results. With the consideration of this, GA method is introduced to optimize the parameter values of SVM.

In this paper, OP2S 1-D simulation model is built and validated by experiment. The results that calculated by 1-D simulation model are used as training samples. After the predicting model established, the optimized scavenging parameters are found with the optimizing goal of IMEP.

\section{OP2S Configuration}

The OP2S diesel engine configuration is shown in Fig. 1 and the structural parameters of OP2S are illustrated in Table 1.

Two pistons are opposed put in the horizontal cylinder. Intake and exhaust ports are respectively distributed on two sides of the cylinder. Rockers are placed on either ends of the cylinder body; the upper end of the rocker is connected to the piston by a small connecting rod, and the lower end of the rocker is connected to the crankshaft by a big connecting rod. The reciprocating movements of the pistons are driven by crankshaft through the rockers and connecting rods. Intake ports are used to deliver fresh air into cylinder, and the exhaust ports are used to deliver burned gas out from cylinder. When two pistons achieve punch movement and the positions are inside inner-side of the ports, the ports are sealed by pistons. When two pistons achieve reverse movement and move to the outside of the inner-side of the ports, the ports are unsealed. Fresh charge goes through intake ports, the burned gas in cylinder is replaced. In order to make sure there is a phase difference between intake piston and exhaust piston, asymmetric angle is implemented. [10]

From Fig. 1, we can see the height and width of intake and exhaust ports can influence the air flowrate. So they are vital factors in designing scavenging system. Crank asymmetric angle $(\gamma)$ can influence the scavenging duration time through influencing the piston motion. [10]So it should also be taken into account.

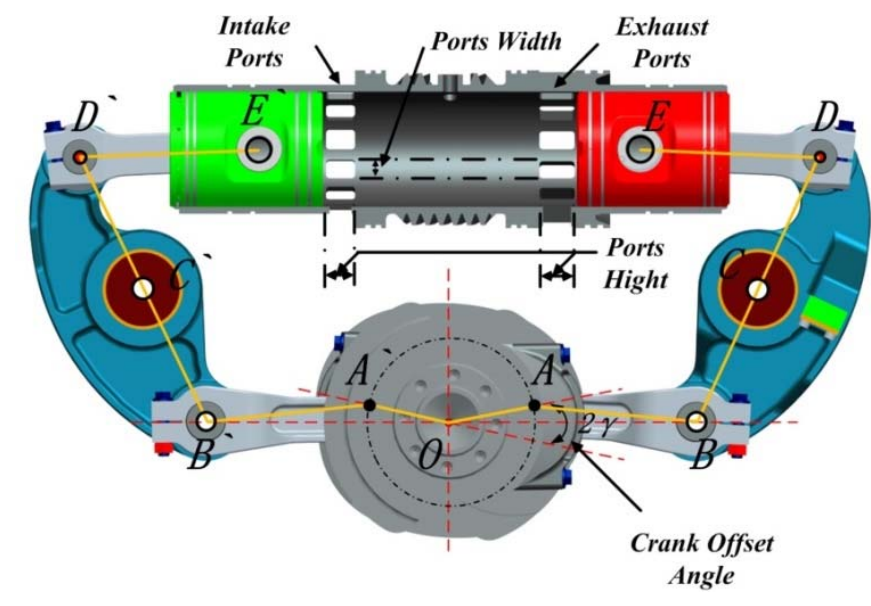

Fig. 1 scavenging system of OP2S diesel engine

Table 1 structural parameters of OP2S diesel engine

\begin{tabular}{|l|l|}
\hline Parameters & Value \\
\hline Bore/mm & 100 \\
\hline Stroke/mm & 110 \\
\hline $\begin{array}{l}\text { Geometric compression } \\
\text { ratio }\end{array}$ & 22 \\
\hline $\begin{array}{l}\text { Practical compression } \\
\text { ratio }\end{array}$ & 15.8 \\
\hline Rated power/Kw & $170 /(2500 \mathrm{r} \cdot \mathrm{min}-1)$ \\
\hline Maximum torque/N·m & $776 /(1600 \mathrm{r} \cdot \mathrm{min}-1)$ \\
\hline $\begin{array}{l}\text { Effective } \\
\text { displacement/L }\end{array}$ & 2.6 \\
\hline
\end{tabular}

\section{Definition of Scavenging Parameters}

As described in the former section, there are five parameters (intake ports' length and width, exhaust ports ' length and width and crank asymmetric) influences the scavenging process of OP2S diesel engine. It is necessary to make definitions of these parameters. 
The intake ports length is defined as

$$
\alpha_{i}=\frac{h_{s}}{S} \in(0.10 \sim 0.12)
$$

Where $\alpha_{i}$ is nondimensionalized intake ports length; $h_{s}$ is intake ports length; $S$ is piston stroke; the value range is $0.1-0.12$.

The intake ports width is defined as

$$
\beta_{i}=\frac{b_{s}}{\pi D} \in(0.70 \sim 0.80)
$$

Where $\beta_{i}$ is nondimensionalized intake ports width; $b_{s}$ is intake ports width; $D$ is cylinder diameter; the value range is $0.70-0.80$.

The exhaust ports length is defined as

$$
\alpha_{e}=\frac{h_{e}}{S} \in(0.123 \sim 0.141)
$$

Where $\alpha_{e}$ is nondimensionalized exhaust ports length; $h_{e}$ is exhaust ports length; $S$ is piston stroke; the value range is $0.123-0.141$.

The exhaust ports width is defined as

$$
\beta_{e}=\frac{b_{e}}{\pi D} \in(0.60 \sim 0.70)
$$

Where $\beta_{e}$ is nondimensionalized exhaust ports width; $b_{e}$ is exhaust ports width; $D$ is cylinder diameter; the value range is $0.60-0.70$

The crank asymmetric angle range is defined as

$$
\gamma_{n} \in\left(5^{\circ} \sim 15^{\circ}\right)
$$

Where $\gamma_{n}$ is crank asymmetric angle, the value range is $5^{\circ}-15^{\circ}$.

\section{1-D Model Establishment}

OP2S 1-D simulation model is established by GT-Power because of its flexibility [12,13]. As Fig.2 shows, there are six subsystems which are intake system, electric auxiliary booster system, intercooler system, OP2S diesel engine system and exhaust system in the model. Two main differences from conventional engine model should be illustrated.

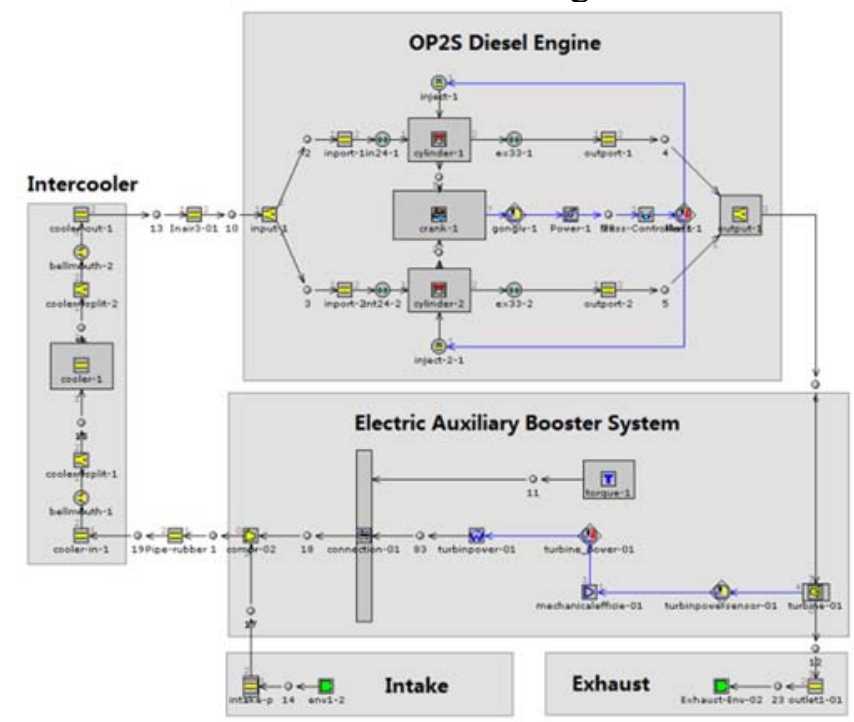

Fig.2 OP2S diesel engine 1-D model

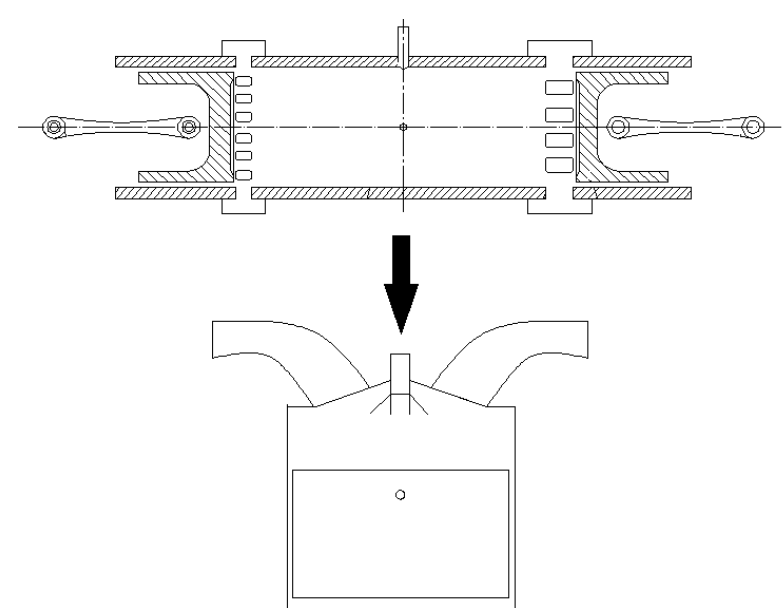

Fig.3 OP2S diesel engine traditional engine translation

Since there is no specific model for OP2S engine, equivalently convert opposed engine model to 
traditional engine model should be done as Fig. 3 shows. Define top dead center as the nearest position of intake piston and exhaust piston. Input piston displacement data is achieved by adding the value of two pistons` placement as Fig.4 shows.

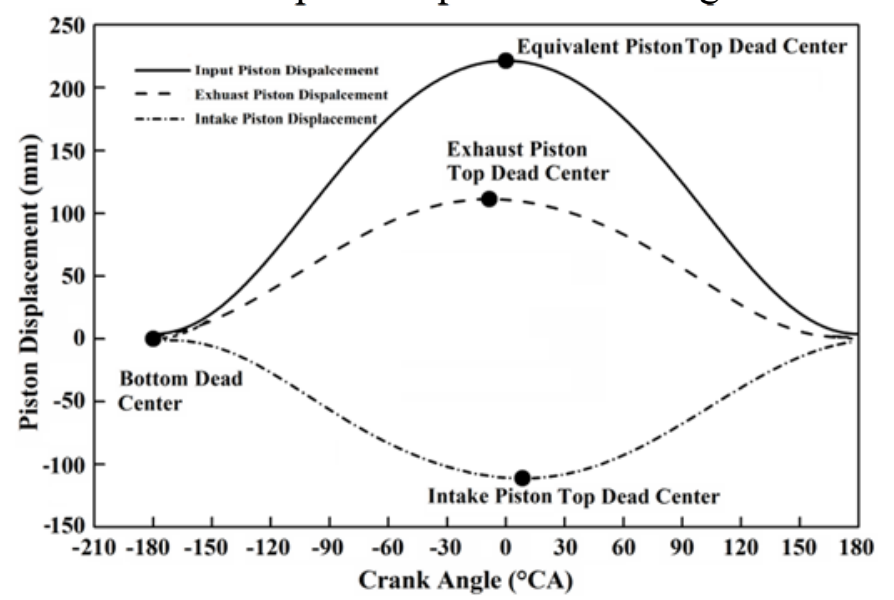

Fig.4 Input piston placement curve

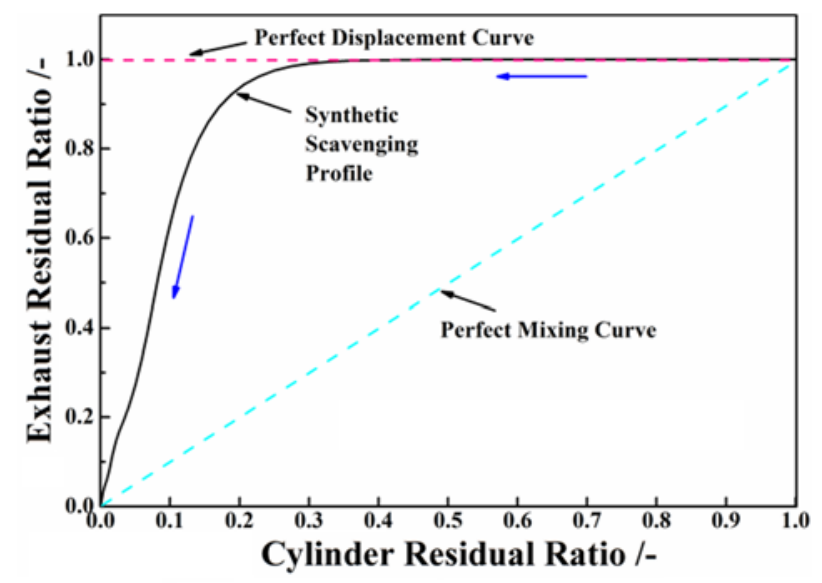

Fig.5 The synthetic scavenging profile of OP2S diesel engine

Scavenging process is also a vital part in the model because in two stroke engines, replacement and mixture between fresh gas and burn gas exit at same time. A lot of two stroke scavenging models have been proposed to express this complex phenomenon $[14,15,16,17,18]$. Among these models, the synthetic profile model for overall characterization of the scavenging process is widely used in engineering and scientific research [19,20,21,22,23]. The work of obtaining the synthetic scavenging profile has been illustrated in literature [24]as Fig.5 shows. The x-axis represents the ratio of the burned gas to all the gas in cylinder. The y-axis represents the ratio of the burned gas to all the gas in exhaust manifold. The profile should be analyzed with the guide of blue arrow., the cylinder and exhaust chamber are filled with burned gas. So both cylinder residual ratio and exhaust chamber residual ratio remain 1. After the scavenging process begins, the fresh charge flows into cylinder. The fresh air goes into the cylinder through intake ports, part of the burned gas is replaced by the fresh air. So the exhaust residual ratio remains 1 while the cylinder residual ratio decreased to 0.35. After the fresh air is short circuited, some fresh air goes into exhaust chamber. So the exhaust residual ratio decreased while cylinder residual ratio decreases. This profile will be used as boundary condition of 1D simulation.

\section{1-D Model Validation}

The experimental system is built by three sub-systems including control system, OP2S diesel engine system and data acquisition system as Fig.6 shows. The control system is based on control base platform to control the operating parameters such as fuel injection timing, rail pressure, engine speed and so on. The OP2S diesel engine operating conditions can be adjusted by dynamometer. Kistler 6065A pressure sensor is used to measure the cylinder pressure and kistler 2614B angle instrument is applied to measure crankshaft signal. The experimental and simulation result under the condition of 1.3bar intake pressure, 1600rlmin (full load), 1bar exhaust pressure is shown in Fig.7. From Fig.7 we can see that the simulation data has good agreement with experimental data. The 1-D model established by the former part is reasonable and can be used to carry out the following work. 


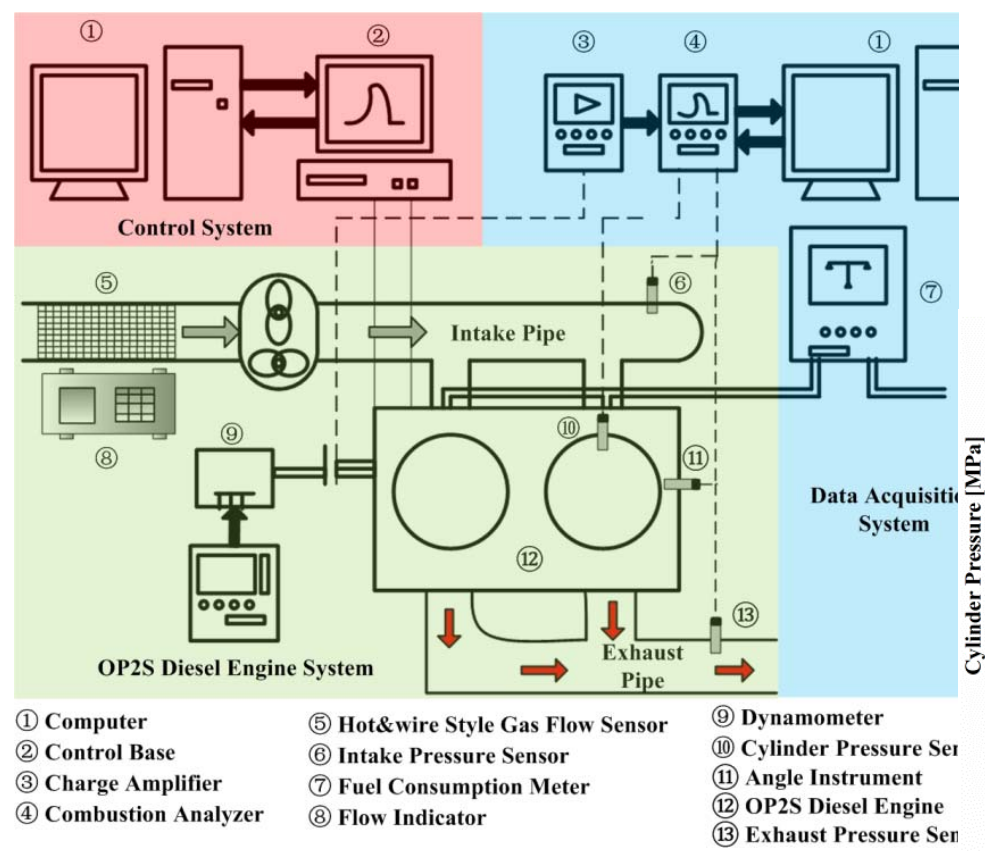

Fig.6 Sketch of OP2S diesel engine experiment system

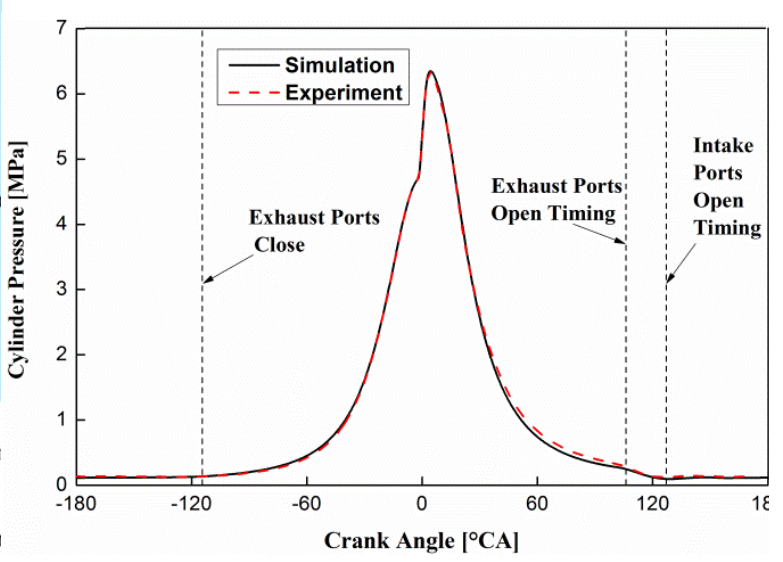

Fig.7 Validation of Cylinder Pressure

\section{GA-SVM Model Setup \\ Overall Description of GA-SVM Model Setup Process}

The overall description of GA-SVM model setup process is shown in Fig. 8. The yellow part is to organize samples. The rad part and green part are to build GA-SVMs for different input samples. The blue part is to select a final predicting model with the standard of minimum Mean Square Error (MSE).

The calculating samples are designed by the combination of the scavenging parameters. The samples are divided into ten parts. Select one of the parts as validation samples and the rest parts are treated as training samples. The training samples are used to train the GA-SVM prediction model and the validation samples are utilized to check the accuracy of the predicting model. Through the method described above, ten SVM predicting models can be established.

The rad area and green area are respectively reflects the process of SVM method and GA. Two parameters, penalty factor $(C)$ and slack variable $(\xi)$, are vital in deciding the accuracy of SVM prediction model. GA is used to optimize the two parameters mentioned above. The converging principle in genetic algorithm is the error between generation and next generation is less than $1 \%$.

The final predicting model is selected with the principle of the minimum error from the ten predicting models that have been established. 


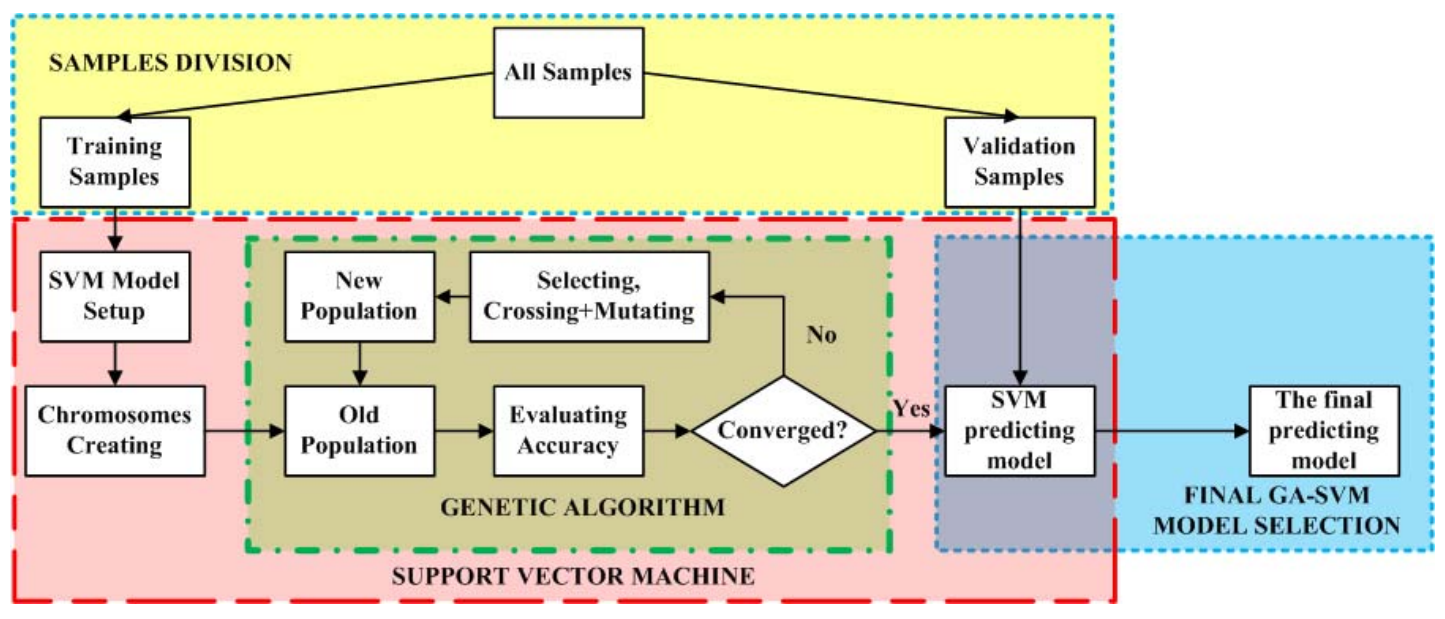

Fig.8 Overall description of GA-SVM model setup process

\section{Training Datum Set Up}

The definition and value range of scavenging parameters are referenced in the former section. For each scavenging parameter, take five values within the value range. Since there are five parameters that can influence the scavenging process of OP2S diesel engine, there will be 3125 calculating cases. With the principle of exhaust ports area should be larger than intake ports area, 1325 cases are removed and 1800 calculating cases are left. Divide these 1800 calculating cases into ten groups. There are 180 cases in one group. For the first SVM prediction model, take the first group as validation group and the rest groups are acted as training groups. For the second SVM prediction model, take the second group as validation group and so forth. Ten predicting models can be established and take the predicting model with minimum error as predicting tool in the next step.

\section{Principle of SVM}

SVM is a regression analysis method based on statistical learning theory proposed by Vapnik [25]. It is widely used in statistic prediction through training a certain amount of training datum. For the training datum $\left\{\left(x_{1}, \mathrm{y}_{1}\right),\left(x_{2}, \mathrm{y}_{2}\right), \cdots,\left(x_{i}, \mathrm{y}_{i}\right) \subset \mathrm{R}\right\}$, The goal is to find a function $f(x)$ to obtain targets $y_{i}$ for all the training datum $x_{i}$ that has the minimum deviation. The function $f(x)$ can be chosen with the form as linear function

$$
f(x)=\omega \cdot x+b
$$

Where $\omega$ is weight vector and $b$ denotes the bias.

Vapnik introduced the slack variables $\xi, \xi_{i}^{*}$ [26] and the problem can be formulated as

$$
\begin{cases}\min & \frac{1}{2}\|\omega\|^{2}+C \sum\left(\xi_{i}+\xi_{i}^{*}\right), \\ \text { s.t. } & y_{i}-\omega \cdot x-b \leq \varepsilon+\xi_{i}, \\ & \omega \cdot x+b-y_{i} \leq \varepsilon+\xi_{i}^{*}, \\ & \xi_{i} \geq 0, \quad \xi_{i}^{*} \geq 0,\end{cases}
$$

Where $\mathrm{C}$ is the penalty factor that determining the tradeoff between the empirical risk and regularization term. $\xi_{i}, \xi_{i}^{*}$ are two slack variables which measure the deviation $\left(y_{i}-f\left(x_{i}\right)\right)$ from the boundaries of the insensitive zone $(\varepsilon)$.

A standard dualization method utilizing Lagrange multipliers [27] is applied to solve this problem like following 


$$
\begin{cases}\max & -\frac{1}{2} \sum_{i, j=1}^{n}\left(\alpha_{i}-\alpha_{i}^{*}\right)\left(\alpha_{j}-\alpha_{j}^{*}\right) \mathrm{K}\left(\chi_{i}, \chi_{j}\right)+\sum_{i=1}^{n} \mathrm{y}_{i}\left(\alpha_{i}-\alpha_{i}^{*}\right)-\varepsilon \sum\left(\alpha_{i}+\alpha_{i}^{*}\right), \\ \text { s.t. } & \sum_{i=1}^{n}\left(\alpha_{i}-\alpha_{i}^{*}\right)=0 \\ & 0 \leq \alpha_{i}, \quad \alpha_{i}^{*} \leq C\end{cases}
$$

Where $\alpha_{i}$ and $\alpha_{i}^{*}$ are Lagrange multiplier coefficients; $\mathrm{K}\left(\chi_{i}, \chi_{j}\right)$ is the kernel function, the most used kernel functions are including linear kernel function, polynomial kernel function, REF kernel function and Sigmoid kernel function. REF kernel function is used in this paper.

Therefore, through the equations above, we can get

$$
\omega=\sum_{i=1}^{n}\left(\alpha_{i}-\mathrm{a}_{i}^{*}\right) x_{i}
$$

For the parameter b in Eq.6, Karush-Kuhn-Tucker(KKT)conditions [28,29] is exploited. Thus, b is decided by the following inequalities [30]

$$
\max \left\{-\varepsilon+\mathrm{y}_{i}-\omega \cdot x_{i} \mid \alpha_{i}<\mathrm{C} \quad \text { or } \quad \alpha_{i}^{*}>0\right\} \leq b \leq \min \left\{-\varepsilon+\mathrm{y}_{i}-\omega \cdot x_{i} \mid \alpha_{i}>0 \quad \text { or } \quad \alpha_{i}^{*}<C\right\}
$$

Finally, $f(x)$ can be expressed by

$$
f(x)=\sum_{i=1}^{n}\left(\alpha_{i}-\alpha_{i}^{*}\right) x_{i} \cdot x+b
$$

\section{Genetic Algorithm}

Genetic Algorithm proposed by Professor J. Holland is a self-adaption global optimization probability search algorithm based on the biological natural selection and genetics basis [31]. Each gene represents each variable. Chromosome (Sk) is composed by multi-genes and the individual is characterized by its chromosomes. Through the recombination among individuals from one generation to next generation, based on the principle of survival of the fittest $\mathrm{f}(\mathrm{Sk})$, high fitness individuals are selected.

As mentioned in the former part, two vital parameters influence the accuracy of the SVM predicting model. The goal of GA method is to find an optimized penalty factor (C) and slack variable $(\xi)$. GA parameters are listed in Table 2 . Individuals are evaluated based on the following function

$$
F(m)=\frac{1}{n} \sum_{i=1}^{n}\left(a_{i}-e_{i}\right)^{2}
$$

Where $a_{i}$ and $e_{i}$ are respectively actual values and estimated values of $i^{\text {th }}$ training sample. Low value of Eq. 12 is corresponding to high fitness.

Table 2 parameters values of GA

\begin{tabular}{|l|l|}
\hline Parameters & Value \\
\hline Evolutionary algebra & 200 \\
\hline Crossover probability & 0.7 \\
\hline Mutation probability & 0.1 \\
\hline Range of penalty factor & {$[0.1,30]$} \\
\hline $\begin{array}{l}\text { Range of radial basis } \\
\text { parameter }\end{array}$ & {$[0.1,30]$} \\
\hline
\end{tabular}

\section{GA-SVM prediction model selection}

Ten predicting models based on GA-SVM method are established and the mean square errors (MSE) 
of these ten models for predicting the validation group are listed in Table 3. The model with minimum MSE can be used in the optimizing process. As we can see in Table 3 the seventh model can be used as predicting model for the next part.

Table 3 parameters and MSE in different predicting models

\begin{tabular}{|c|c|c|c|}
\hline $\begin{array}{l}\text { Predicting } \\
\text { model }\end{array}$ & $\begin{array}{l}\text { Penalty factor in } \\
\text { SVM }\end{array}$ & $\begin{array}{l}\text { Slack variable in } \\
\text { SVM }\end{array}$ & $\begin{array}{l}\text { MSE for predicting validation } \\
\text { group }\end{array}$ \\
\hline 1 & 4.978 & 25.5589 & 0.0059 \\
\hline 2 & 9.2941 & 22.2677 & 0.0062 \\
\hline 3 & 7.3923 & 23.1519 & 0.0071 \\
\hline 4 & 6.3599 & 24.4474 & 0.0068 \\
\hline 5 & 8.0796 & 21.1708 & 0.0064 \\
\hline 6 & 8.0748 & 21.4451 & 0.0067 \\
\hline 7 & 7.3792 & 25.9265 & 0.0058 \\
\hline 8 & 8.3556 & 23.7638 & 0.0076 \\
\hline 9 & 14.8389 & 19.6501 & 0.0051 \\
\hline 10 & 3.7005 & 25.2116 & 0.0065 \\
\hline
\end{tabular}

\section{Optimization Process and Results}

The predicting model for OP2S diesel engine IMEP has been established in the sections above. Based on the predicting model, the optimizing process is shown in Fig.9. After the calculation of the cases, find the case with highest IMEP. Compare the IMEP with the former highest IMEP, if the error is less than $1 \%$, it is converged. Otherwise, decrease the value step and make new combination of scavenging parameters until finding the optimized scavenging parameters combination.

The optimized scavenging parameters are listed in Table 4 and the predicted IMEP and the IMEP calculated by GT-Power are also included.

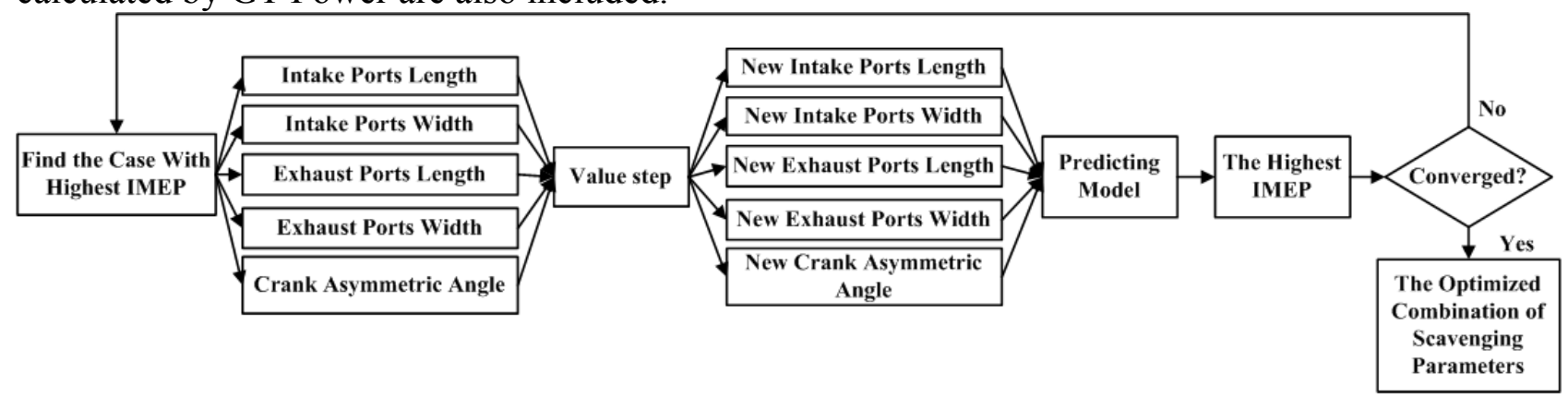

Fig.9 GA-SVM model optimization process

Table 4 optimized scavenging parameters and results

\begin{tabular}{|l|l|}
\hline Parameters & Value \\
\hline$h_{i}$ & 0.104 \\
\hline$\beta_{i}$ & 0.771 \\
\hline$h_{e}$ & 0.1229 \\
\hline$\beta_{e}$ & 0.667 \\
\hline$\gamma\left[^{\circ}\right]$ & 8.43 \\
\hline Prediction IMEP [bar] & 12.91 \\
\hline Simulation IMEP [bar] & 12.86 \\
\hline
\end{tabular}




\section{Conclusions}

(1) Since there is no model for OP2S diesel engine in GT-Power, some equivalents is necessary. Piston displacement curve and the synthetic scavenging profile are vital in modelling OP2S diesel engine GT-Power model.

(2) GA-SVM with 1-D software is an effective and rapid method in predicting and optimizing. It is also available in OP2S diesel engine scavenging system design.

(3) Trough GA-SVM, the optimized parameters are found. Though the prediction result and the simulation result have deviation, the final result is satisfied.

\section{Acknowledgments}

This work is supported by the National Ministry Fundamental Research Foundation of China (grant no. B2220110005) and the BIT Foundation for Fundamental Research (Grant No. 20120342015).

\section{References}

[1] Flint M, Pirault J P. Opposed Piston Engines: Evolution, Use, And Future Applications [J]. SAE International, Warrendale, PA ISBN, 1-15.

[2] Naik, S., Johnson, D., Koszewnik, J., Fromm, L. et al., "Practical Applications of Opposed-Piston Engine Technology to Reduce Fuel Consumption and Emissions," SAE Technical Paper 2013-01-2754, 2013,1-15.

[3] Regner, G., Herold, R., Wahl, M., Dion, E. et al., "The Achates Power Opposed-Piston Two-Stroke Engine: Performance and Emissions Results in a Medium-Duty Application," SAE Int. J. Engines 4(3):2726-2735, 2011, 1-10.

[4] Herold, R., Wahl, M., Regner, G., Lemke, J. et al., "Thermodynamic Benefits of Opposed-Piston Two-Stroke Engines," SAE Technical Paper 2011-01-2216, 2011, 1-15.

[5] Redon, F., Kalebjian, C., Kessler, J., Rakovec, N. et al., "Meeting Stringent 2025 Emissions and Fuel Efficiency Regulations with an Opposed-Piston, Light-Duty Diesel Engine," SAE Technical Paper 2014-01-1187, 2014, 1-10.

[6] Regner, G., Johnson, D., Koszewnik, J., Dion, E. et al., "Modernizing the Opposed Piston, Two Stroke Engine for Clean, Efficient Transportation," SAE Technical Paper 2013-26-0114, 2013, 1-13.

[7] Franke, M., Huang, H., Liu, J., Geistert, A. et al., "Opposed Piston Opposed Cylinder (opoc) 450 hp Engine: Performance Development by CAE Simulations and Testing," SAE Technical Paper 2006-01-0277, 2006,1-10.

[8] Hofbauer, P., "Opposed Piston Opposed Cylinder (opoc) Engine for Military Ground Vehicles," SAE Technical Paper 2005-01-1548, 2005, 1-43.

[9] Zhang Z, Zhao C, Wu D, et al. Effect of Piston Dynamic on the Working Processes of an Opposed-Piston Two-Stroke Folded-Cranktrain Engine[R]. SAE Technical Paper, 2014,1-10.

[10]Rainer J. ROTHBAUER, Roland H. MARGELIK, Muhammad M. ASLAM, "Predictive Simulation Strategies for the 2-Stroke Scavenging Process within the Scope of the Development Process," SAE Technical Paper 2005-32-0099, 2005,1-17.

[11]N. Cristianini, J. Shawe-Taylor, Support Vector Machines and other Kernel-Based Learning Methods, Cambridge University Press, 2000,1-20.

[12]Morel, T., Keribar, R., and Leonard, A., "“Virtual Engine/Powertrain/Vehicle" Simulation Tool Solves Complex Interacting System Issues," SAE Technical Paper 2003-01-0372, 2003, 1-13.

[13]Ciesla, C., Keribar, R., and Morel, T., "Engine/Powertrain/Vehicle Modeling Tool Applicable to 
All Stages of the Design Process," SAE Technical Paper 2000-01-0934, 2000, 1-13.

[14]Benson R S, Brandham P T, A method for obtaining a quantitative assessment of the influence of charging efficiency on two-stroke engine performance, International Journal of Mechanical Sciences, 1969, 11(3): 303-312.

[15]Dang D, Wallace F J, Some single zone scavenging models for two-stroke engines, International journal of mechanical sciences, 1992, 34(8): 595-604.

[16] Sher E, Harari R, A simple and realistic model for the scavenging process in a crankcase-scavenged two-stroke cycle engine, Proceedings of the Institution of Mechanical Engineers, Part A: Journal of Power and Energy, 1991, 205(2): 129-137.

[17] Streit E E, Borman G L, Mathematical simulation of a large turbocharged two-stroke diesel engine, SAE Technical Paper, 1971,1-11.

[18]Wallace, F. and Cave, P., Experimental and Analytical Scavenging Studies on a Two-Cycle Opposed Piston Diesel Engine, 1971, SAE Technical Paper 710175,1-12.

[19]Bozza, F. and Gimelli, A., A Comprehensive 1D Model for the Simulation of a Small-Size Two-Stroke SI Engine, 2004, SAE Paper 2004-01-0999,1-15.

[20]Ferrara, G., Balduzzi, F., and Vichi, G., An Innovative Solution for Two-Stroke Engines to Reduce the Short-Circuit Effects, 2012, SAE Technical Paper 2012-01-0180,1-12.

[21]Laget, O., Ternel, C., Thiriot, J., Charmasson, S. et al., Preliminary Design of a Two-Stroke Uniflow Diesel Engine for Passenger Car, SAE Int. J. Engines 6(1), 2013,pp.596-613.

[22] Tribotte P, Ravet F, Dugue V, et al, Two Strokes Diesel Engine-Promising Solution to Reduce CO2 Emissions, Procedia-Social and Behavioral Sciences, 2012, 48: 2295-2314.

[23]Pohorelsky, L., Brynych, P., Macek, J., Vallaude, P. et al.: Air System Conception for a Downsized Two-Stroke Diesel Engine, 2012, SAE Technical Paper 2012-01-0831,1-14.

[24]Liu Y, Zhang F, Zhao Z, et al. Study on the synthetic scavenging model validation method of opposed-piston two-stroke diesel engine[J]. Applied Thermal Engineering, 2016, 104: 184-192.

[25]Vapnik V N. An Overview Of Statistical Learning Theory[J]. Neural Networks, IEEE Transactions On, 1999, 10(5): 988-999.

[26] Vapnik, the nature of statistical learning theory. Springer, new York,pp.281-304.

[27]Fletcher R. Practical methods of optimization john wiley \& sons[J]. New York, 1987, 80.

[28]Karush W. Minima of functions of several variables with inequalities as side conditions[D],1939.

[29]Kuhn, H W and A W Tucker. Nonlinear programming, 1951. Proc 2nd Berkeley symposium on mathematical statistics and probabilistics, Berkeley, university of California press, pp 481-492.

[30]Keerhi, S.S. improvements to platti`s SMO algorithm for SVM classifierdesign. Technical report CD-99-14, Dept. of mechanical and production engineering, Natl. Univ. Singapore.singapore.

[31]Goldberg D E. Genetic Algorithms In Search, Optimization And Machine Learning[M].New York:Addison-Wesley,1989. 